\title{
Modeling and simulation of transients in electric power systems using hybrid system theory
}

\author{
Yury Shornikov*, Evgeny Popov \\ Automated Control Systems Department, Novosibirsk State Technical University, 630073, 20 Prospekt K. Marksa, Novosibirsk, Russia
}

\begin{abstract}
Transients in electric power systems are of great interest to power engineers when designing a new or maintaining an existing system. The paper deals with using hybrid system theory for modeling and simulation of an electric power system with controllers. The presented technique is rather convenient and recommended as mathematical models of transients in electric power systems with controllers in general contain both continuous and discrete components. The modeling and simulation were carried out in the modeling and simulation environment ISMA, which is briefly presented in the paper.
\end{abstract}

\section{Transients in electric power systems}

An electric power system (EPS) [1] is a network of elements generating, transforming (transformers, voltage stabilizers, power invertors), transmitting and distributing (power lines, feeders), and consuming (loads) electrical energy along with different controllers and protective devices.

Transients might occur in an EPS due to faults or during its normal functioning. The examples are a threephase ground fault caused by a fallen tree and change of the loads as a result of, for example, a start of an asynchronous motor at a factory, which are rather common. In a transient state, the currents, voltages and other system characteristics change over time. States of this kind are not preferred by the users since they expect the system properties to be rather constant, i.e. the power line frequency and voltage magnitude in their electrical outlets to be about, for example, $50 \mathrm{~Hz}$ and $220 \mathrm{~V}$ respectively. Components of the second type, controllers and protective devices, are intended for preventing the damage or malfunction of the electrical elements by making transients less destructive, bringing the system to a quasi-steady state.

\section{Hybrid systems}

Although electrical components are described by differential-algebraic systems of equations (DAEs), that is their models are continuous, elements of the second type are characterized as having discontinuities, more precisely the corresponding DAEs' right-hand sides have discontinuities. Models of this sort can be conveniently described as hybrid systems (HS) [2, 3] exhibiting both discrete and continuous behaviors. An HS is a generalization of dynamical system and can be regarded as a sequence of classical dynamical systems (modes) activated one after another so the final conditions of one dynamical system are the initial conditions for the next one [4].

$$
\text { A tuple } \quad<j, y^{j}, f^{j}, y_{0}^{j}>, j \in\left\{1 . . n_{m}\right\} \text {, }
$$

$f_{j}: R \times R^{N_{j}} \rightarrow R^{N_{j}}$, where the mode behavior $y^{j}(t) \in R^{N_{j}}$, is called an HS mode; the mode function $f^{j}$ satisfies the Lipschitz conditions [5] for all $\left(t, y^{j}\right) \in R \times R^{N_{j}}$.

A continuously differentiable function $g(y, t): R \times R^{N} \rightarrow R^{s}, \quad s=1,2, \ldots$ is referred to as an event function and is related to the predicate $p r: g(y, t)<0, p r \in B=\{$ false, true $\}$.

An event function $g^{j}\left(y^{j}, t\right)$ of a mode behaves at all of the mode solutions in such a manner that the corresponding predicate $p r_{j}: g^{j}\left(y^{j}, t\right)<0$ of the mode is true ( $p r_{j}=$ true $)$ at the half-open interval of the mode solution $\left[t_{j}^{0}, t_{j}^{*}\right) \subseteq\left[t_{0}, t_{k}\right]$.

The event surface of an HS mode is the boundary $G \subset R^{n}$ where the event function $g(y, t)=0$.

An HS event is the HS state in time and space $(y, t) \in R^{n+1}$ when the continuously differentiable function $g(y, t): R \times R^{n} \rightarrow R^{s}$ reaches the event surface.

Modes are directly related to events and are their consequences. An event can appear instantly as a result of changing various hybrid system characteristics: parameters, initial conditions, continuous states and their derivatives (the right-hand sides of systems of first order ordinary differential equations), physical properties of the hybrid system.

A very elementary and classic example of a hybrid system, the bounce of a perfectly elastic ball off a perfectly elastic rigid surface, can be described with the following initial value problem:

* Corresponding author: shornikov@inbox.ru 


$$
\begin{gathered}
\frac{d y}{d t}=v_{y}, \frac{d v_{y}}{d t}=-g, \\
y(0)=h, v_{y}(0)=0, \\
\text { if }(y \leq 0) \text { and }\left(v_{y} \leq 0\right) \\
\text { then } v_{y}=-v_{y} .
\end{gathered}
$$

An HS mode can be constrained by one or more conditions $(s \geq 1)$. In our case, the mode of falling down to the bounce surface corresponds to the simultaneous fulfillment of the following two conditions: the vertical velocity is negative $g_{1}\left(v_{y}, t\right)=v_{y}<0$; the distance to the bounce surface is positive, so $g_{2}(y, t)=-y<0$.

As events (transitions of an HS from one mode to another) may dramatically change the behavior of the hybrid system, their detection during the simulation is rather a crucial task. Even small errors can accumulate causing the visible change of the trajectory behavior. Failures to detect events may lead to even much worse simulation results having nothing to do with the system model.

\section{Modeling transients in electric power systems}

Let us consider the following electric power system, a six-machine test system of Institute "Energosetproekt" (Russia) [6]. Its one line diagram is shown in Figure 1.

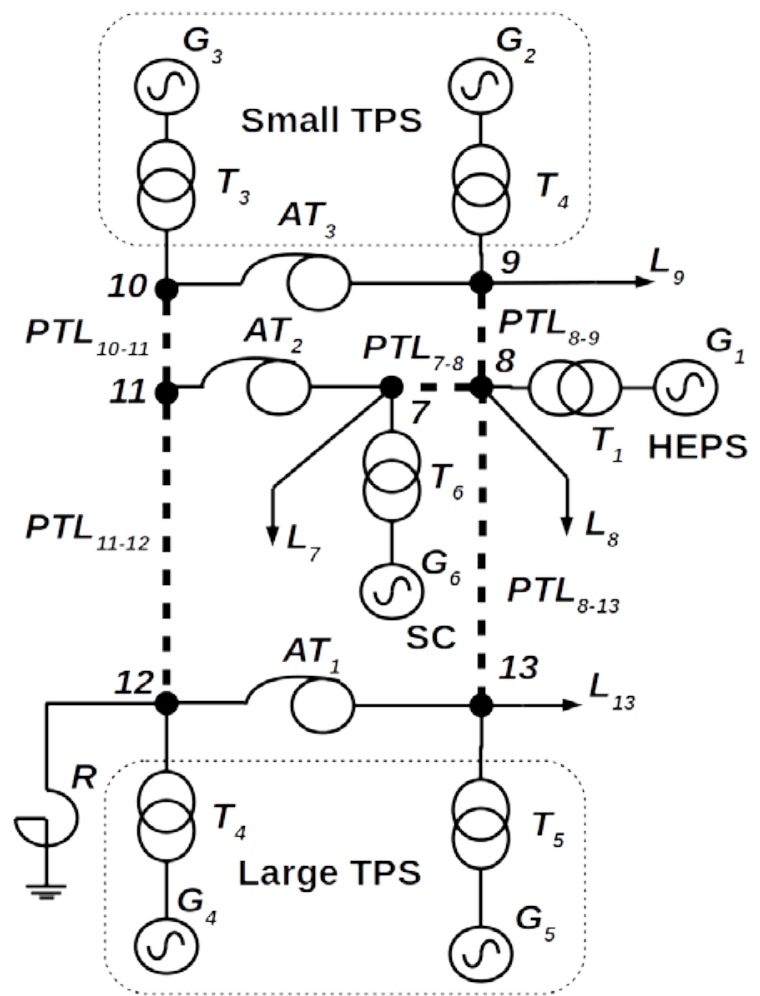

Fig. 1. One-line diagram.

The system consists of six synchronous generators $\left(G_{1}-\right.$ $\left.G_{6}\right)$, the corresponding generator transformers $\left(T_{1}-T_{6}\right)$, three autotransformers $\left(A T_{1}-A T_{3}\right)$, four loads modeling the power consumers, five power transmission lines denoted by bold dashed lines, and a current limiting reactor $(R)$.

The voltage rating of the power transmission lines $P T L_{7-8}, P T L_{8-9}$, and $P T L_{8-13}$ is $220 \mathrm{kV}$, whereas that of $P T L_{10-11}$ and $P T L_{11-12}$ is $500 \mathrm{kV} . G_{1}$ models a powerful hydroelectric power station (HEPS). The couples $G_{2}, G_{3}$ and $G_{4}, G_{5}$ represent a small and a large thermal power stations (TPS) respectively. The generator $G_{6}$ models the synchronous compensators (SC) installed at a transmission substation.

After applying Park's transformation [7], in the corresponding $d-q$ rotating reference frame, generators without damper windings are described as

$$
\begin{gathered}
\frac{d \psi_{d}}{d t}=-u_{d}-\omega \psi_{q}-r_{a} i_{d}, \\
\frac{d \psi_{q}}{d t}=-u_{q}+\omega \psi_{d}-r_{a} i_{q}, \\
\frac{d \psi_{f}}{d t}=E_{q e} \frac{r_{f}}{x_{a d}}-r_{f} i_{f}, \\
\psi_{d}=\frac{1}{\omega_{0}}\left(x_{d} i_{d}+x_{a d} i_{f}\right), \\
\psi_{q}=\frac{1}{\omega_{0}} x_{q} i_{q}, \\
\psi_{f}=\frac{1}{\omega_{0}}\left(x_{f} i_{f}+x_{a d} i_{d}\right), \\
\frac{d \omega}{d t}=\frac{1}{T_{J}}\left(M_{T}-M\right), \\
M=i_{q} \psi_{d}-i_{d} \psi_{q},
\end{gathered}
$$

where $\psi_{d}, \psi_{q}$ are the projections of the flux linkages of the stator windings on the axes $d$ and $q$ respectively; $r_{a}$ is the resistance of the stator windings; $u_{d}, u_{q}$ and $i_{d}, i_{q}$ stand for the stator winding voltage and current projections respectively; $\omega$ denotes the angular speed of the rotor; $\omega_{0}$ is the rated power line frequency; $\psi_{f}$ is the field winding flux linkage; $E_{q e}$ is the induced electromotive force; $x_{d}$ and $x_{q}$ stand for the direct and quadrature synchronous reactances respectively; $x_{f}$ is the field winding reactance; $r_{f}$ denotes the field winding resistance; $i_{f}$ is the field winding current; $x_{a d}$ stands for the direct stator reaction inductance; $T_{J}$ is the inertia constant; $M_{T}$ is the turbine torque; $M$ is the electric torque.

All the generators are equipped with automatic voltage regulators, which stabilize the output voltage as the loads change or a fault occurs. The generators $G_{1}-$ $G_{5}$ have turbine speed controllers maintaining the angular speeds of the rotors as close to the rated one as possible since even pretty small variations can lead to the damage of the synchronous machines and other components of the network.

The block diagram for the turbine speed controllers of the generators $G_{2}$ and $G_{3}$ is shown in Fig. 2. 


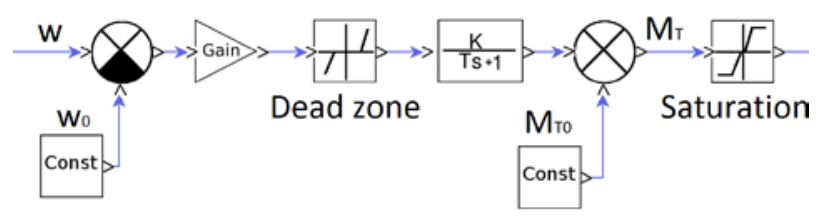

Fig. 2. Block diagram of the turbine speed controller.

Given the angular speed, the controller computes the displacement of the centrifugal pendulum clutch having a dead zone. The output signal, turbine torque, is bounded to an upper and a lower limits by the saturation block.

The controller can be represented in the LISMA language [8] as shown in Fig. 3.

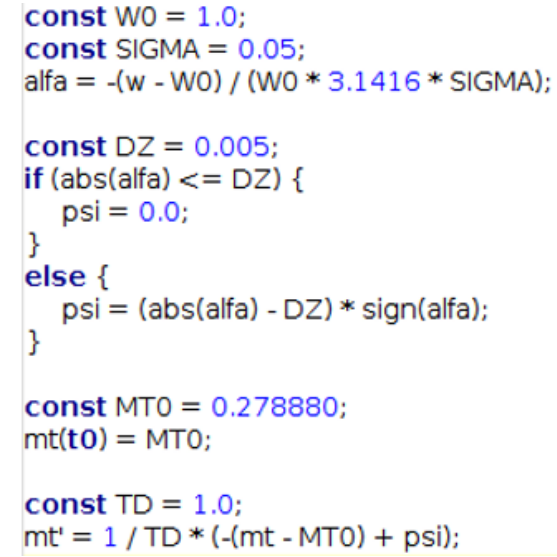

Fig. 3. Textual model of the turbine speed controller.

As long as we use Park-Gorev's equations, all the other equations have to be mapped onto the $d$ - $q$ frame of a reference synchronous machine [6, 7]. It results in a system of equations of coordinate transformation in the form of

$$
\begin{gathered}
\tilde{i}_{d j}=i_{d j} \cos \delta_{j}+i_{q j} \sin \delta_{j}, \\
\tilde{i}_{q j}=-i_{d j} \sin \delta_{j}+i_{q j} \cos \delta_{j}, \\
\tilde{u}_{d j}=u_{d j} \cos \delta_{j}+u_{q j} \sin \delta_{j}, \\
\tilde{u}_{q j}=-u_{d j} \sin \delta_{j}+u_{q j} \cos \delta_{j}, \\
\frac{d \delta_{j}}{d t}=\omega_{r}-\omega_{j} .
\end{gathered}
$$

where $\delta_{j}$ stands for the angle between the rotors of synchronous machine $j$ and the reference synchronous machine; $\tilde{i}_{d j}, \tilde{i}_{q j}, \tilde{u}_{d j}, \tilde{u}_{q j}$ are the projections of the current and voltage of machine $j$ onto the axes of the reference synchronous machine. System of equations (3) is written for every synchronous machine in an EPS but the reference one.

Transformers and autotransformers can be represented as active-inductive elements as shown in Fig. 4 a.

The power transmission lines are modeled as activeinductive elements with two active-capacitive connections to ground. The equivalent circuit is depicted in Fig. 4 b.

One of the simplest equivalent circuits for loads is an active-inductive element connected to ground (Fig. 5 a).
Reactors can be represented in the same way but with resistances of zero (Fig. 5 b).

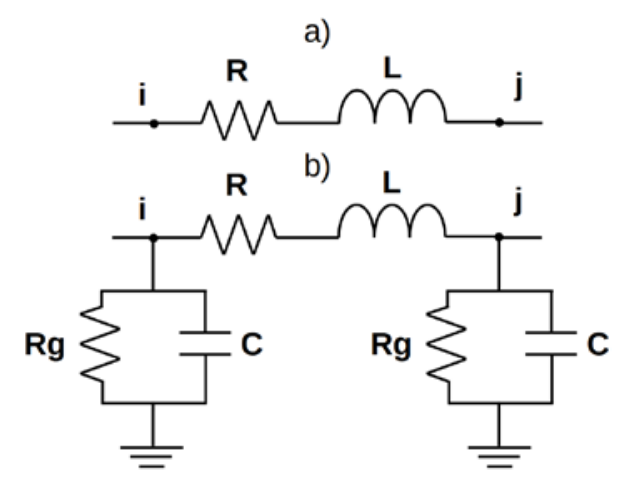

Fig. 4. Equivalent circuits for a) transformers and autotransformers, b) power transmission lines.

In order to obtain the full model, we also need to include a system of equations of current conservation according to Kirchhoff's first law.

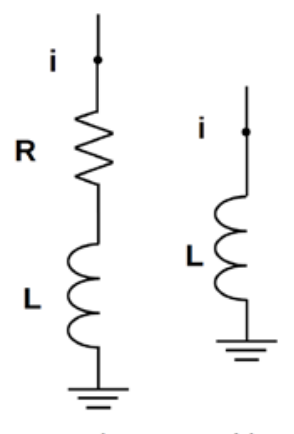

a) b)

Fig. 5. Equivalent circuits for a) loads, b) reactors.

The full system of equations contains 144 differential and 65 algebraic equations. The given models allow us to study both electromechanical and electromagnetic transient phenomena.

\section{Modeling and simulation environment ISMA}

ISMA [8] (the acronym for "Computer-aided analysis tools" in Russian) is a modeling and simulation environment developed by the Automated Control Systems department of Novosibirsk State Technical University (Russia).

ISMA supports several input modeling languages, including the textual general-purpose modeling language LISMA, a graphical language of block diagrams, and a graphical language for modeling electric power systems (Fig. 6).

It allows one to model EPSs using the aforementioned component models, carry out simulations with different scenarios including changes in the system parameters, short circuits, line breaks.

In ISMA's EPS editor, controllers of generators as well as protective devices can be conveniently modeled using block diagrams. The model in Fig. 2 was created in ISMA’s block diagram editor. 
Events in HSs can be classified into three groups: unilateral, bilateral, and accuracy critical events [4]. The division is necessary because it is impossible to accurately detect a switching point as phase space trajectories are computed using finite precision arithmetic. The problem of detection of the time instant $t=t^{*}$, when an event function $g(y(t), t)=0$, is actually a complex problem of accurate event detection $[9,10$, $11]$.

If there are singularities or the physical meaning of a problem imposes the condition that the phase space trajectories must not ever cross the event surface, then such cases fall into the group of unilateral events. What is exactly the case in the presented model since the controllers have dead zones, the turbine powers are bounded as well as the rate at which the controllers can change them.

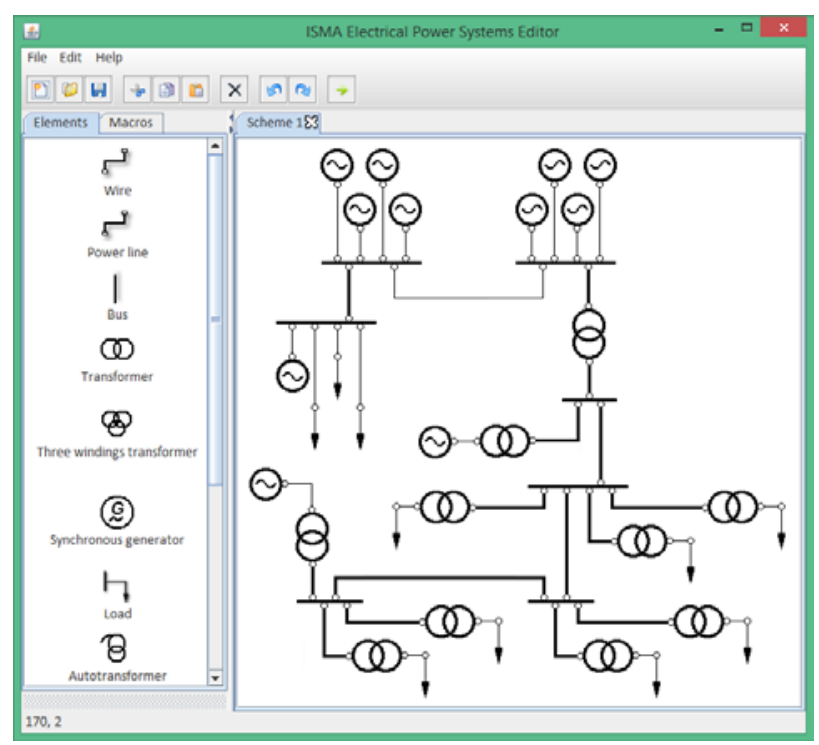

Fig. 6. ISMA's EPS editor.

For event detection ISMA employs an original event detection algorithm based on the ideas of J. Esposito [8, 9], which allows correct detection of events. The algorithm is based on the following theorem.

Theorem. Computing the step size for an explicit numerical method of the form $y_{n+1}=y_{n}+h_{n+1} \varphi_{n}$ by using the formula

$$
h_{n+1}=(\gamma-1) g_{n}\left(\frac{\partial g_{n}}{\partial y} \varphi_{n}+\frac{\partial g_{n}}{\partial t}\right)^{-1}, \gamma \in(0,1),
$$

guarantees the event dynamics to behave as a stable linear system, which approaches the surface $g(y, t)=0$. Besides, if $g\left(y_{0}, t_{0}\right)<0$, then $g\left(y_{n}, t_{n}\right)<0$ for all $n$.

Also, a distinctive feature of ISMA is that it allows one to use one-step explicit numerical methods with improved stability regions and stability control [12] for simulating moderately stiff [13] systems of equations, whereas it is a common practice to employ implicit methods for this purpose [14, 15].
Let the load at Node 8 decrease by $20 \%$ at $t=0 \mathrm{~s}$. The reaction of the electric torque of the nearest generator $G_{1}$ is shown in Fig. 7. All the values are given in a per-unit system [7]. Apart from the electromagnetic transient, the event results in a much longer lasting electromechanical transient (Fig. 8).

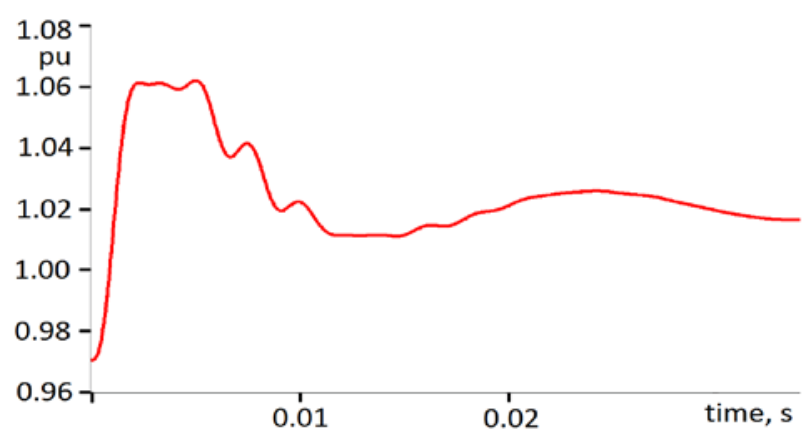

Fig. 7. Course of $M_{G 1}$

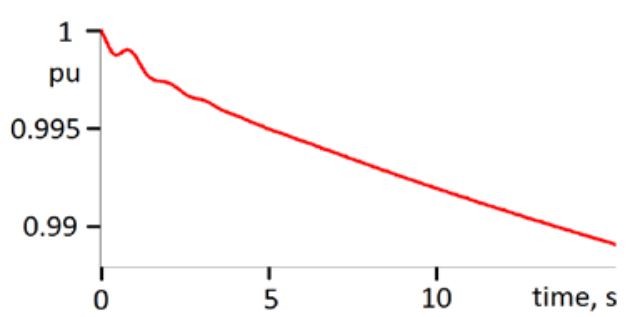

Fig. 8. Course of $\omega_{G 1}$

Now let us suppose that, at $t=0.1 \mathrm{~s}$, there happens a three-phase ground fault at Node 12 . The duration of the fault is $0.05 \mathrm{~s}$. The simulation results are shown in Figures 9 and 10.

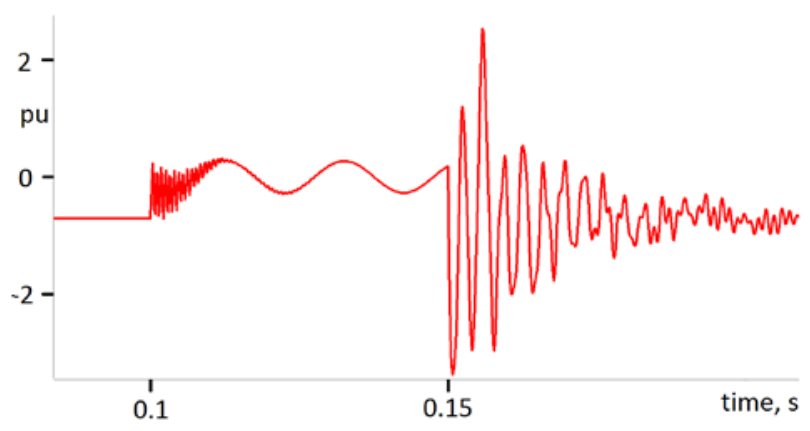

Fig. 9. Course of $u_{d G 4}$.

The modeling and simulation results show that hybrid system theory can be successfully applied to solving certain power engineering problems, simulating transients in electric power systems. ISMA's Electric Power System Editor facilitates the proposed techniques.

\section{Simulation}




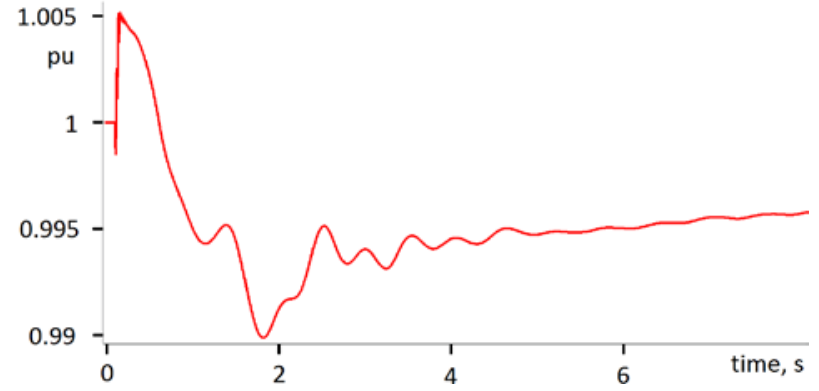

Fig. 10. Course of $\omega_{G 4}$.

This work was supported by the grant of the Russian Foundation for Basic Research (RFBR grant 17-07-01513) and by the grant of the Education, Audiovisual and Culture Agence (EU), programme ERASMUS+ Capacity building in higher education, project 573751-EPP-1-2016-1-DE-EPPKA2-CBHEJP, Innovative teaching and learning strategies in open modelling and simulation environment for student-centered engineering education.

\section{References}

1. A. von Meier. Electric power systems. A conceptual introduction (John Wiley \& Sons, Inc., Hoboken, New Jersey, 2006)

2. P.J. Antsaklis, A brief introduction to the theory and applications of hybrid systems, Proceedings of the IEEE 88, No.7, pp.879-887 (2000)

3. A. J. van der Schaft, H. Schumacher, An introduction to hybrid dynamical systems (Lecture Notes in Control and Information Sciences 251, 2000)

4. J.M. Esposito, Simulation and control of hybrid systems with applications to mobile robotics. Dissertation (USA, 2002)

5. E. Hairer, S. Nørsett, G. Wanner, Solving Ordinary Differential Equations I: Nonstiff Problems ( Springer, 2011)

6. T. Yu. Fomina, Razrabotka algoritma raschjota pjerjehodnyh protsjessov slozhnyh rjegulirujemyh EES: dis... kand. tehn. nauk (Development of an algorithm for simulating transients in complex controlled electric power systems. Candidate of technical sciences dissertation) (Russia, 2014) (in Russian)

7. V.A. Venikov, Transient phenomena in electrical power systems (Pergamon press, 1964)

8. Yu. V. Shornikov, D. N. Dostovalov, Features of the ISMA modeling and simulation environment, Proceedings of International multi-conference on engineering, computer and information sciences (SIBIRCON), P. 332-337 (2017)

9. J.M. Esposito, V. Kumar, A state event detection algorithm for numerically simulating hybrid systems with model singularities, ACM Transactions on Modeling and Computer Simulation, vol. 17, iss. 1, p. 1-22 (2007)

10. C.W.Gear, O.Osterby, Solving ordinary differential equations with discontinuities, ACM transactions on mathematical software 10(1), p. 23-44 (1984)
11. L.F.Shampine, I.Gladwell, R.W.Brankin, Reliable solution of special event location problems for ODEs, ACM transactions on Mathematical Software 17(1), p. 11-25 (1991)

12. E. A. Novikov, M. A. Rybkov, Application of explicit methods with extended stability regions for solving stiff problems, Mathematics \& Physics 9 (2), p. 209-219 (2016)

13. E. Hairer, G. Wanner, Solving Ordinary Differential Equations II: Stiff and Differential-Algebraic Problems (Springer, 2011).

14. F.E. Cellier, E. Kofman, Continuous system simulation (Springer, 2006)

15. Yu.V. Shornikov, B.U. Uatay, E.A. Novikov, Methods for Solution of Stiff Problems (KazakhBritish Technical University, 2010). 